\title{
Unusual cause of an abduction deficit in a 14 year old girl
}

\author{
M A S Ahmed, A Powell, R Borgstein, L Alsford
}

Answers on p 799.

King George Hospital, Ilford, London, UK M A S Ahmed

\section{North Middlesex} Hospital Trust, Edmonton, London, UK

A Powell

R Borgstein

L Alsford

Correspondence to: Dr M A S Ahmed, Paediatric Department, King George Hospital, Barley Lane, Goodmayes, Ilford, Essex IG3 8YB, UK mas.ahmed@ rbhc-tr.n.thames.nhs.uk

A 14 year old girl presented with sudden onset of diplopia and slurred speech with no fever. A week previously, she had a headache, cough, and rhinorrhoea. However her symptoms resolved a few days before admission. Eye examination confirmed an abduction paresis of the left eye and abduction nystagmus of the right eye. Esotropia of the left eye was absent when the eyes were fixated in the primary position. There was full vertical eye movement, insufficient vestibulo-ocular reflex, and slow saccades of the affected eye. Neurological evaluation revealed slurred speech and ataxic gait.

Cerebrospinal fluid (CSF) analysis showed 40 white cells (all lymphocytes), protein of $0.4 \mathrm{~g} / \mathrm{l}$, and normal glucose content. Viral serology and bacterial cultures of blood, urine, CSF, stools, and throat swab were negative. Polymerase chain reaction analysis was negative for herpes simplex genome in the CSF. Serological tests for toxoplasmosis and Mycoplasma pneumoniae were negative. An autoimmune screen and a metabolic profile were normal. An electroencephalogram showed bilateral delta waves, more posteriorly, with no epileptiform discharges.

Magnetic resonance imaging (MRI) of the brain using T2 weighted axial images (fig 1) showed an increased signal in the cerebellar white matter and the dorsal aspect of the lower pons. There was an increased signal in the region of the abducens nucleus and the paramedian pontine reticular formation.

Treatment with oral erythromycin, intravenous acyclovir, and cefotaxime was started immediately and a degree of clinical improvement was seen within 48 hours. After three weeks of treatment, ocular movement, speech, and gait returned to normal and a repeat CSF analysis showed no abnormality. Subsequent MRI of the brain showed a marked improvement in the abnormal areas identified previously (fig 2).

\section{Questions}

(1) What is the most likely diagnosis?

(2) Give possible differential diagnoses of an abduction deficit.

(3) How would you manage a patient with an abduction deficit?

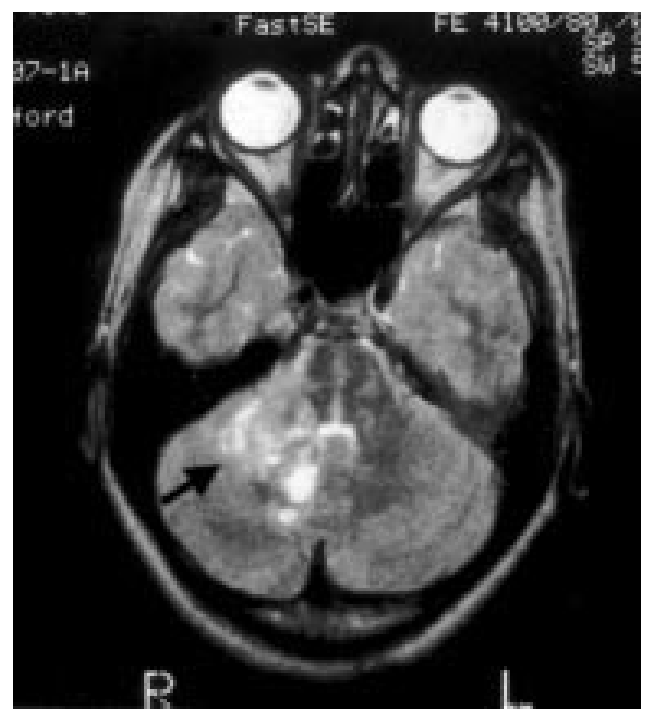

Figure 1 T2 weighted axial image showing increased signal in the cerebellar white matter. There is increased signal at the dorsal aspect of the lower pons, and lateral to this on the left at the level of paramedian pontine reticular formation and abducens nucleus.

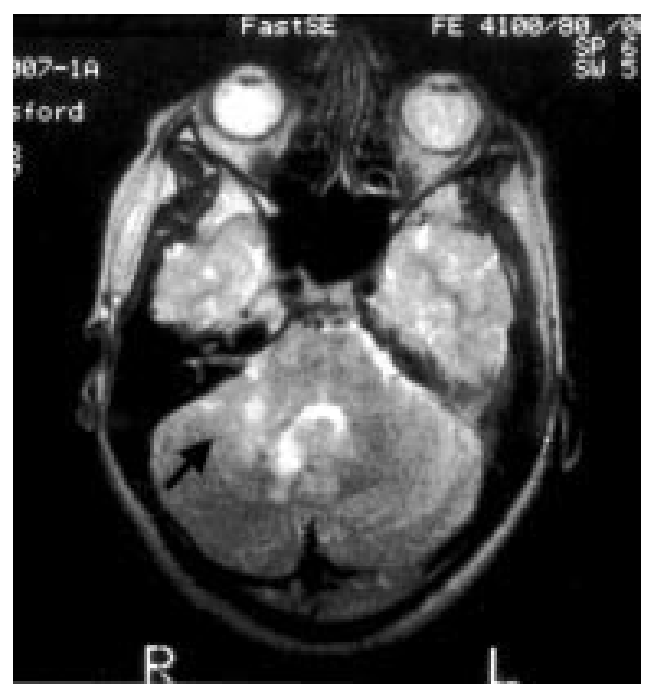

Figure $2 \quad T 2$ weighted axial image showing a marked improvement in the previously identified abnormal areas of high signal. 


\section{An unusual cause of acute bacterial meningitis}

I Stephenson, M J Wiselka

A 31 year old man was admitted after a 24 hour febrile illness with headache and confusion. On examination he was unwell, pyrexial at $39.4^{\circ} \mathrm{C}$, tachycardic, and uncooperative. He became increasingly confused and agitated and exhibited signs of meningism. There was no rash or focal localising neurological signs, and visual fields to confrontation were normal.

He required intubation and sedation to control his agitation and was admitted to the intensive care unit. Lumbar puncture revealed turbid cerebrospinal fluid (CSF) containing $8300 \times 10^{6} / 1$ white blood cells $(95 \%$ polymorphs), protein $4.6 \mathrm{~g} / \mathrm{l}$, glucose $1.4 \mathrm{mmol} / \mathrm{l}$

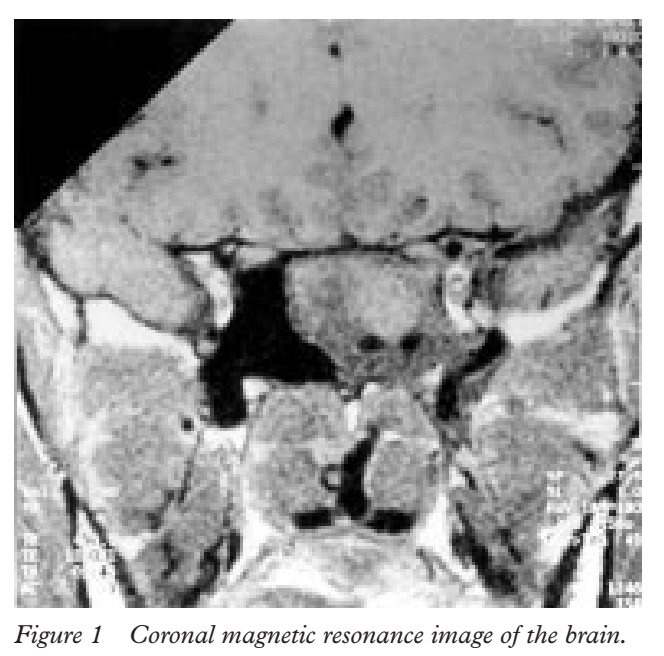

(blood glucose $5.9 \mathrm{mmol} / \mathrm{l}$ ), with negative Gram stain and culture. Blood and throat cultures were sterile. Nasal swabs cultured methicillin resistant Staphylococcus aureus. Computed tomography of his head with contrast showed expansion of the sella and generalised cerebral oedema. Further imaging with magnetic resonance and coronal computed tomography of the pituitary fossa was obtained (fig 1). Thyroid function, random cortisol, follicle stimulating hormone, and luteinising hormone were normal but raised values of prolactin (15400 IU/1) and decreased testosterone concentrations $(9.0 \mathrm{nmol} / \mathrm{l})$ were found.

He received two weeks of intravenous ceftriaxone, vancomycin, and metronidazole and made an uncomplicated recovery. CSF rhinorrhoea was noted by the patient on bending forwards. Subsequent CSF cultures, obtained from the rhinorrhoea, was sterile.

Department of Infectious Diseases and Tropical Medicine, Leicester Royal Infirmary, Leicester LE1 5WW, UK

I Stephenson

M J Wiselka

Correspondence to:

Dr Stephenson

istephen@globalnet.co.uk

Submitted 8 September

2000

Accepted 6 November 2000
Figure 1 Coronal magnetic resonance image of the brain.

\section{Questions}

(1) What is the lesion shown on the magnetic resonance imaging scan?

(2) How does this usually present?

(3) How may this condition present with meningism?

(4) What is the treatment of the underlying lesion?

(5) What are the causes of hyperprolactinaemia? 